\title{
Computer Optimized Design of Electron Guns
}

\author{
John David * Lawrence Ives $^{\dagger}$ Hien Tran * Thuc Bui ${ }^{\dagger}$ \\ Michael Read ${ }^{\dagger}$
}

June 28, 2007

\begin{abstract}
This paper considers the problem of designing electron guns using computer optimization techniques. Several different design parameters are manipulated while considering multiple design criteria including beam and gun properties. The optimization routines are described. Examples of guns designed using these techniques are presented. Future research is also described.
\end{abstract}

\section{Introduction}

Electron guns are used in many vacuum electron devices to convert electrical power into an electron beam. Electron beam devices include RF sources for numerous applications such as communications, radar, industrial heating, and high energy accelerators. Electron beams are also used in medical and industrial x-ray devices, for electron beam lithograph and electron beam welding, and in cathode ray guns for televisions and oscilloscopes. Many of these devices are critical for national defense and science and industrial applications.

In an RF source, the beam energy is converted to energy in an RF wave. In x-ray sources, welders, or cathode ray devices, precise focusing is required

*Department of Mathematics and Center for Research in Scientific Computation, Box 8205, North Carolina State University, Raleigh, North Carolina 27695-8205, jadavid2@ncsu.edu,tran@ncsu.edu

${ }^{\dagger}$ Calabazas Creek Research, Inc. 690 Port Drive, San Mateo, CA 94404 (650) 312-9575, RLI@CalCreek.com, BUI@Calcreek.com, mike@Calcreek.com 
to obtain high resolution. The electron gun is a primary component in klystrons, traveling wave tubes (TWTs), gyrotrons, and inductive output tubes. The configuration of the gun depends on many factors, including the operating voltage, current, beam size and shape, magnetic focusing circuit, power supply, and operational environment. Consequently, customized electron gun design is required for essentially every new device. Because of the large number of variables, this is often a time consuming and expensive process. Typical design time for a new electron gun for an RF source is 3040 man-hours involving 20-30 design iterations [1]. This is for designs that can be modelled in two dimensions. A number of $2 \mathrm{D}$ simulation tools are available, including EGUN ${ }^{\circledR}$ and TRAK ${ }^{\circledR}$.

The process becomes more demanding for 3D designs. Recent interest in multiple beam and sheet beam guns is placing severe demands on the computational codes as well as the design engineer. 3D analysis is computationally intensive, making iterative design very expensive when performed manually. As an example, CCR recently designed a sheet beam gun for an XBand klystron [2]. The development required approximately 2-3 man-months with approximately 100 iterations, each involving 8 hours of CPU time. The gun operated with Brillouin focusing, so the simulations did not include a magnetic field.

CCR completed the design of an X-Band electron gun using a combination of MAFIA ${ }^{\circledR}$ and another 3D simulation code developed in Russia [3]. This design was performed several years ago and required approximately one year of iterative design. Fortunately, more recent computational tools and experience are reducing this design time, though a recent design of a multiple beam gun for a $200 \mathrm{MHz}$ Multiple Beam Klystron (MBK) still required four months of iterative design [4]. Both these efforts were for singly convergent guns. A recent program to design a doubly convergent multiple beam gun was abandoned because the number of variables and the complexity of the design could not be overcome. This experience provided much of the motivation for developing computer optimized design.

In a typical design process, the engineer begins with an existing configuration close to the new requirement or with design equations for the basic configuration. The design is simulated and geometrical, electrical, and magnetic parameters are modified until the required performance is achieved. In general, the engineer attempts to design an electron gun operating with a specified combination of voltage and current that produces an electron beam of a specific size with laminar electron trajectories. For confined flow guns 
where the magnetic field penetrates to the cathode, the engineer must design both the electrical and magnetic circuits. The requirement is to emit the electrons at a precise angle to the magnetic field to minimize beam ripple [5]. A typical requirement is that the beam envelope not vary more than 5 $\%$ through the device.

In previous research, CCR developed a computer optimization process for Brillouin focused electron guns [6]. This program used EGUN ${ }^{\circledR}$ and MATLAB ${ }^{\circledR}$ routines to produce an electron beam of a specific size at a specified axial location with laminar electron trajectories. This program successfully demonstrated that significant reduction in the design time could be achieved with minimal interaction by the design engineer. This translates into a reduction in design cost and potentially improved performance.

In the current program, CCR is advancing this capability using the $3 \mathrm{D}$ finite element, adaptive mesh code Beam Optics Analysis (BOA). Although the initial designs presented here are two dimensional, the simulations are completely three dimensional. While this is not necessary for these particular designs, it established the process for direct transition to the 3D designs described later. In this program, a confined flow, Pierce electron gun was designed using computer optimization. There were several goals in the development. The initial effort involved modification of simple geometrical parameters to achieve the optimum beam quality. These parameters included the spacing between the cathode and anode to achieve the desired perveance, the relative position of the magnetic circuit to achieve the desired beam compression, and the radius of curvature of the cathode to minimize beam ripple. Figure 1 shows a drawing of the electron gun.

The next task was to demonstrate that nonlinear shaping of electrode surfaces could improve performance. The program focused on two sub tasks. The first was to demonstrate that the shape of the cathode could be optimized to reduce beam ripple while still achieving the desired perveance and beam size. The cathode was defined by a set of points connected with a spline curve. This curve was rotated about the gun axis to define the cathode surface. The second task was to define the focus electrode by a similar set of points and a spline curve, then optimize the shape of the curve to minimize the electric field. Minimization of the electric field will reduce the probability of arcing between the gun and the anode. The final step was to combine these results to demonstrate a high quality electron beam with minimal potential for electrical breakdown. This was followed by application to a sheet beam gun for an X-Band klystron. 


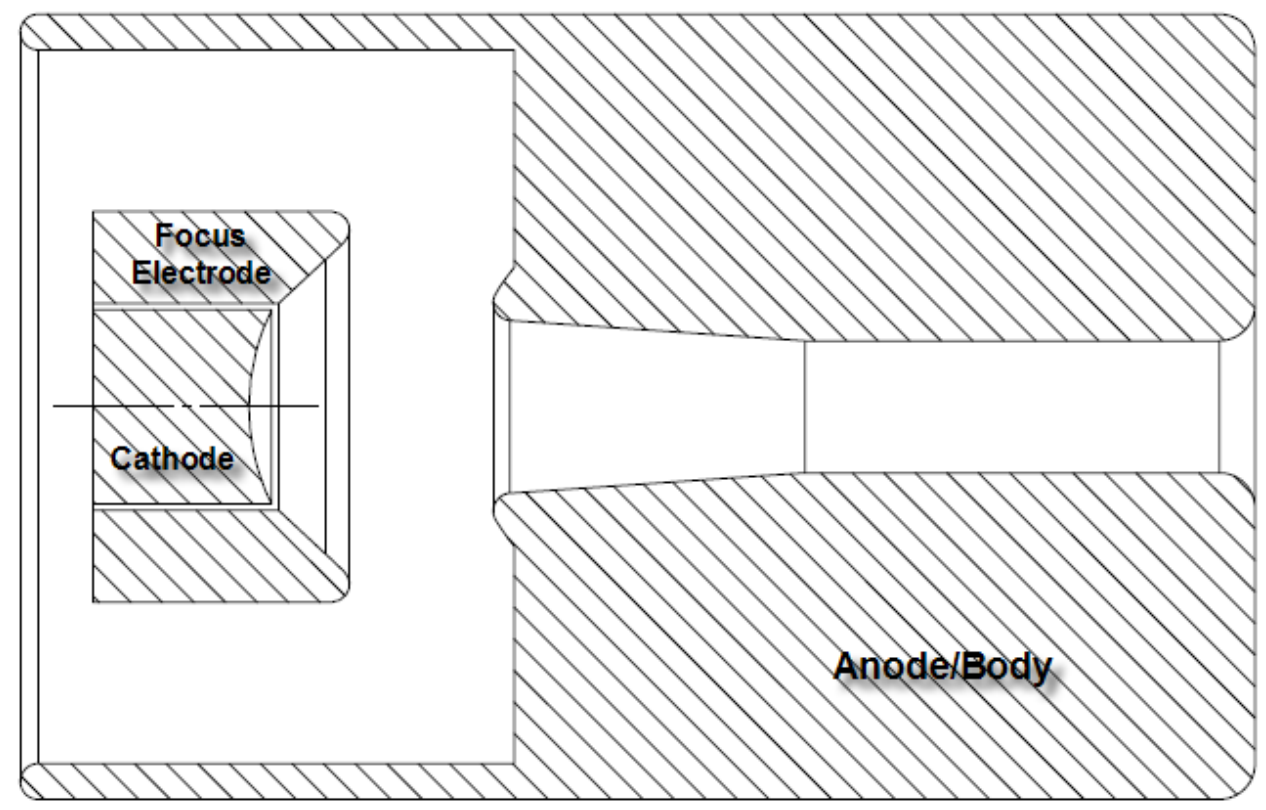

Figure 1: Pierce electron gun.

The organization of the paper is as follows. The gun geometry was generated in SolidWorks ${ }^{\circledR}$ with BOA used for the computer simulations. These tools are described in Section 2. Section 3 describes the process by which MATLAB ${ }^{\circledR}$ routines control the optimization process by executing both SolidWorks ${ }^{\circledR}$ and BOA in batch mode using line commands. Section 4 describes the optimization routines. Sections 5, 6 and 7 describe the simulation results. Section 8 gives a description of the research in progress to extend this development to fully $3 \mathrm{D}$ devices.

Finally, it should be noted that computer optimization is simply a tool. It can not replace the knowledge, experience, and intuition of a trained engineer. Rather it allows the engineer to focus on the physics of the problem while the computer performs the routine, iterative task of parameter variation to achieve the defined engineering goals. 


\section{Computer Tools}

SolidWorks ${ }^{\circledR}$ and BOA are the principal commercial programs used in this research. SolidWorks ${ }^{\circledR}$ is a 3D, parametric, solid modelling program that can generate ACIS-formatted geometry files, a requirement for integration with BOA. Figure 2 shows the Pierce electron gun in SolidWorks ${ }^{\circledR}$. In this figure, the anode/beam tunnel is semi-transparent.

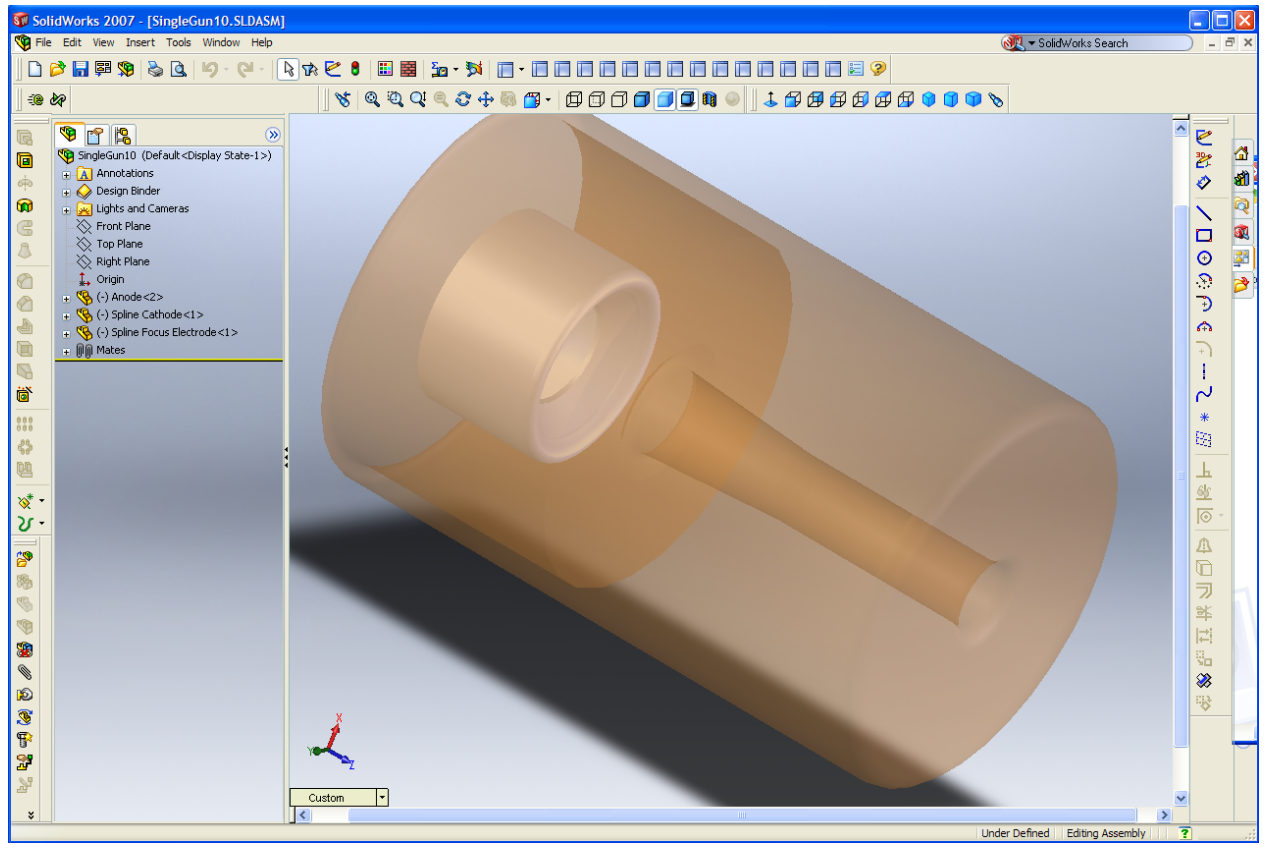

Figure 2: Pierce electron gun in SolidWorks ${ }^{\circledR}$. This is the 3D model of the gun shown in Figure 1. The anode is semi-transparent.

An important feature of parametric modelling is the ability to define key dimensions in design tables. One can then update the geometry by changing values in these tables. This allows an external program to control parametric changes to the geometry. Figure 3 shows a sketch of a spherical cathode in SolidWorks ${ }^{\circledR}$ with the associated design table in Excel ${ }^{\circledR}$. The cathode is created by revolving the sketch about the axis.

SolidWorks ${ }^{\circledR}$ allows batch operation, so the MATLAB ${ }^{\circledR}$ control program can modify the design table, execute the CAD program to generate the updated model, generate the ACIS file, then terminate the CAD program. 


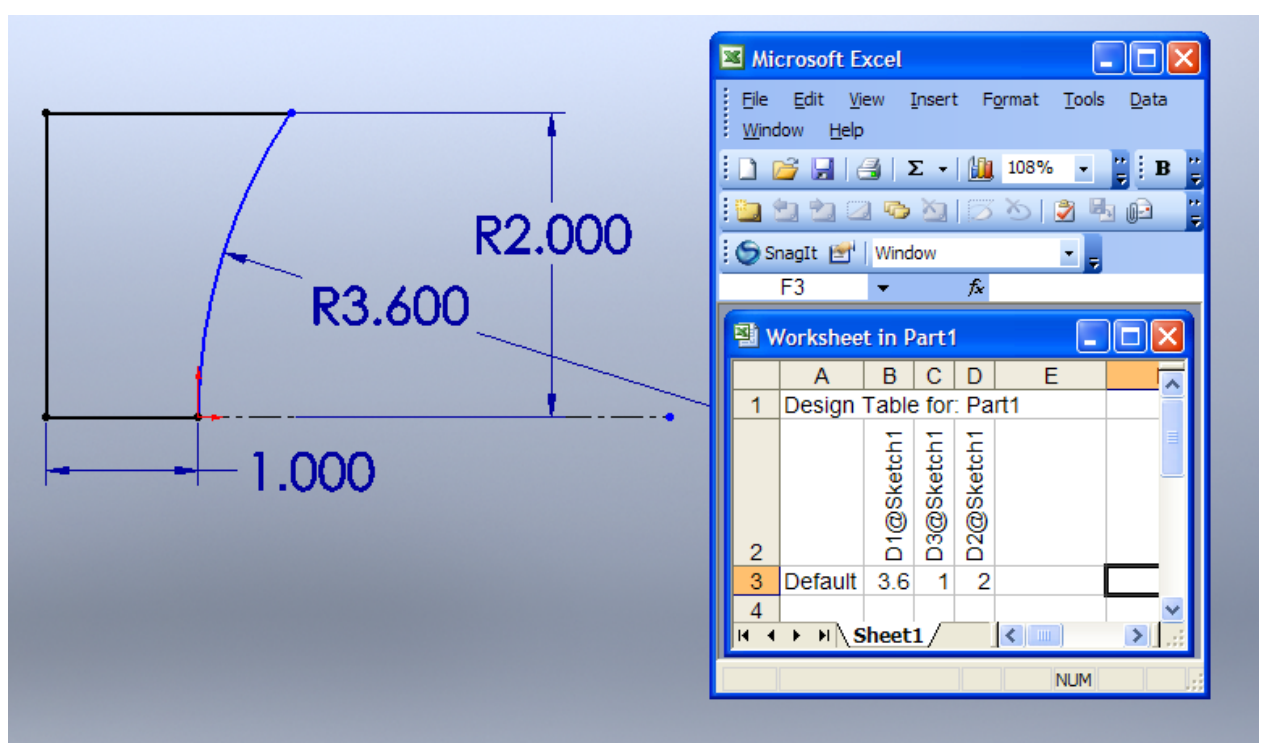

Figure 3: Spherical cathode sketch in Solidworks ${ }^{\circledR}$ with associated design table.

The electron gun simulation is performed using BOA. Like the solid modelling program, BOA can be executed by MATLAB ${ }^{\circledR}$. All input for BOA is contained in an ASCII file that can be modified by the MATLAB ${ }^{\circledR}$ control program. The file contains information for solution of the electric fields, including the voltages assigned to various objects and dielectric constants for ceramics. Electron emitters are also defined using information from the CAD program to identify specific surfaces. The ASCII file provides information controlling the number of trajectories and the temperature and work function for thermionic emitters. All meshing is performed automatically within BOA. For this research, the magnetic field profile was generated by Maxwell $2 \mathrm{D}^{\circledR}$ and used as input to BOA. The axial position of the magnetic circuit relative to the center of the cathode was a variable in the optimization process and controlled the beam compression. Magnetic circuit modelling will soon be implemented within BOA, so input from external programs will not be necessary. This will also allow optimization of the magnetic circuit parameters in future research.

BOA is an adaptive mesh, finite element, 3D analysis code for designing electron beam devices. A principal feature is the adaptive meshing which 
removes the burden for mesh generation from the user and assigns responsibility to the field solver and particle pusher routines. With adaptivity enabled by the user, BOA adapts the mesh density in areas where field gradients are high until the specified accuracy is achieved. It can also coarsen mesh in areas where high accuracy is not required to reduce the computational burden. The user can also control the mesh density in regions occupied by the electron beam and in regions near selected surfaces.

\section{Design Iteration Procedure}

Each iteration of the optimization routine requires several steps. The general process is described in Figure 4, however we will describe each block in more detail here.

As usual, the iterative methods used in this research require a starting point or initial design. For each optimization attempt, the user must specify a set of starting design parameters for the optimization routines. It is generally beneficial if these design parameters are relatively close to the optimal design parameters, however this may not be necessary. There are routines which can consider a general subset of the parameter space and attempt to find a global minimum, but these routines generally require an extensive number of function evaluations, which is not feasible in the case of 3D design.

The first step in a function evaluation is to write the geometry related parameters, e.g., cathode radius or spline parameters, focus electrode shape parameters etc., into Excel ${ }^{\circledR}$ files linked to the SolidWorks ${ }^{\circledR}$ CAD files. The authors used a routine written by Brett Shoelson, which was obtained at MATLAB ${ }^{\circledR}$ central (http://www.mathworks.com/matlabcentral/), an open exchange for MATLAB ${ }^{\circledR}$ users, to write the numerical values from MATLAB ${ }^{\circledR}$ to the Excel ${ }^{\circledR}$ files. SolidWorks ${ }^{\circledR}$ then regenerates the geometry files with the newly updated parameters from the spreadsheets. This produces a geometry file read by BOA, which then executes, producing output files detailing the trajectories of the particles and fields in the electron gun. MATLAB ${ }^{\circledR}$ routines read these files to determine the beam characteristics and calculate a cost function value that measures how well the current design parameters achieve the design goals. Finally, this optimization routine uses this cost function value to either compute a new set of trial design parameters or, in the case that the current design parameters are considered optimal, to terminate the routine. 


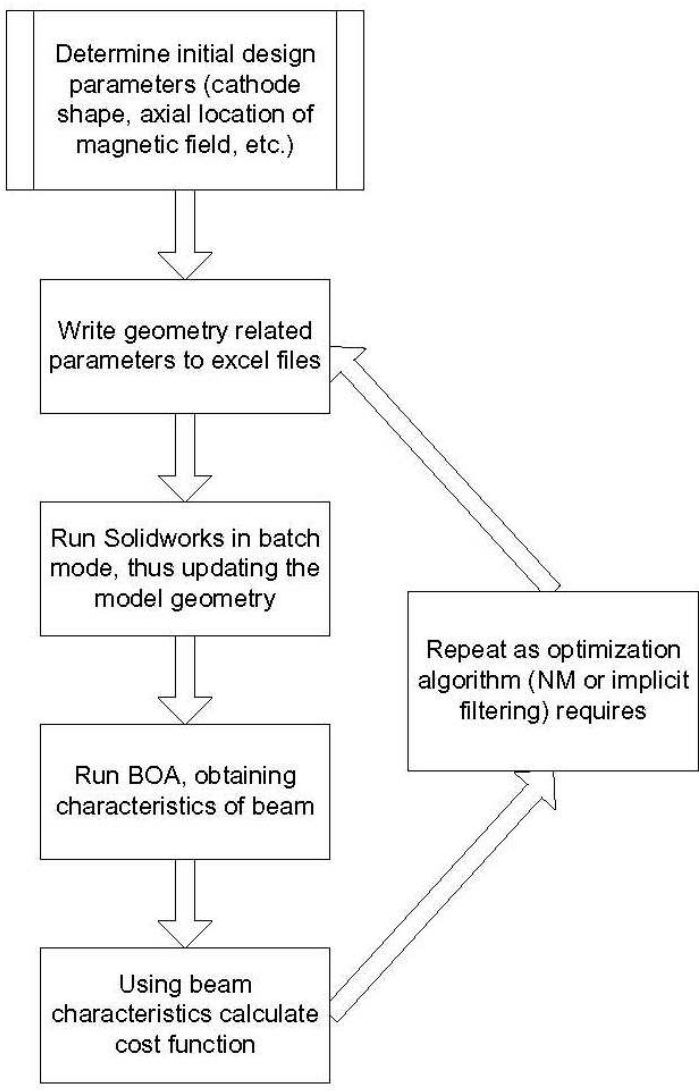

Figure 4: Flowchart for local optimization routine. 


\section{Optimization Routines}

This section provides an overview of the sampling optimization algorithms used in the optimal design of the electron guns. Basically, in an optimal design problem, one begins by formulating a function that characterizes the design goals. The task is then to minimize or maximize this function and thus obtain a design that meets the desired criteria. Mathematically speaking, the problem is given a function $f: \mathbb{R}^{N} \rightarrow \mathbb{R}$ find $\lambda^{*} \in \mathbb{R}^{N}$ such that $f\left(\lambda^{*}\right) \leq f(\lambda)$ for all $\lambda$ of interest. If the $\lambda^{\prime}$ 's of interest are only those near $\lambda^{*}$, then it is a local optimization problem. On the other hand, if the $\lambda$ 's of interest belong to a subset $\Omega \subset \mathbb{R}^{N}$ then it is a global optimization problem. The Nelder-Mead and implicit filtering optimization routines used in this research are known as deterministic sampling methods. Gradient information used by implicit filtering is only approximate, as it is obtained from sampled points in the parameter space. For a discussion on the advantages and disadvantages of sampling based methods versus gradient based methods, the interested reader is referred to [7].

\subsection{Nelder-Mead Algorithm}

The Nelder-Mead algorithm is a deterministic sampling method, i.e., it only requires function values and no gradient information and is thus a simple algorithm to implement. Given $N+1$ points in the available parameter range, it sorts the point such that $J\left(\lambda_{1}\right) \leq J\left(\lambda_{2}\right) \leq \cdots \leq J\left(\lambda_{N+1}\right)$, where $J\left(\lambda_{i}\right)$ is the evaluation of the goal function for parameter $\lambda_{i}$. It then attempts to minimize the function by replacing the point with the highest function value $J\left(\lambda_{N+1}\right)$, which is the worst point, with a point with a lower function value. It first finds the point centered among the other points, not including the worst points $\lambda_{N+1}$

$$
\bar{\lambda}=\frac{1}{N} \sum_{i=1}^{N} \lambda_{i} .
$$

It then attempts to replace $\lambda_{N+1}$ with

$$
\lambda_{N E W}=(1+\beta) \bar{\lambda}-\beta \lambda_{N+1},
$$

where 


$$
\beta=\left\{\beta_{r}, \beta_{e}, \beta_{o c}, \beta_{i c}\right\}
$$

where $\beta$ represents points obtained by reflection $\beta_{R}$, extension $\beta_{E}$, outward contraction $\beta_{O C}$ and inward contraction $\beta_{I C}$ as illustrated in Figure 5 for a $2 \mathrm{D}$ example. If none of these values are better than the previous worst point $\lambda_{N+1}$, the algorithm shrinks the available parameter range toward the best point, i.e., it replaces the point with

$$
\bar{\lambda}_{i}=\frac{\lambda_{i}+\lambda_{1}}{2} .
$$

The algorithm then resorts the points and iterates. Our implementation of the algorithm used the values

$$
\left\{\beta_{r}, \beta_{e}, \beta_{o c}, \beta_{i c}\right\}=\{1,2,1 / 2,-1 / 2\} .
$$

in equation (2).

To better illustrate this method, consider the case when $N=2$. In this case the parameters form a triangle in a 2D plane. Figure 5 illustrates what Nelder-Mead does in this case. Assume $\lambda_{3}$ is the worst parameter, so the algorithm is attempting to replace this point with either a reflection, $r$, expansion, $e$, outward contraction, oc, or inward contraction, $i c$. There are various stopping criteria for this algorithm including iteration number, difference between the function at the best and worst points and the available range of parameters. For a more detailed treatment see [7].

\subsection{Implicit Filtering}

Implicit filtering is a projected quasi-Newton iteration that uses difference gradients, reducing the difference increments as the optimization progresses [8]. The idea is that gradient approximations with a large step size will be insensitive to high-frequency oscillations, which generally produce a large number of local minima, and follow the general landscape of the parameter space. As the routine approaches the minimum, where the oscillations in the parameter space are less, the step size for the gradient is reduced. Figure 6 illustrates a function where implicit filtering may be useful. The specific implementation used in this work is bound constrained, but, for simplicity, the unconstrained version is described. This algorithm optimizes functions of the form 


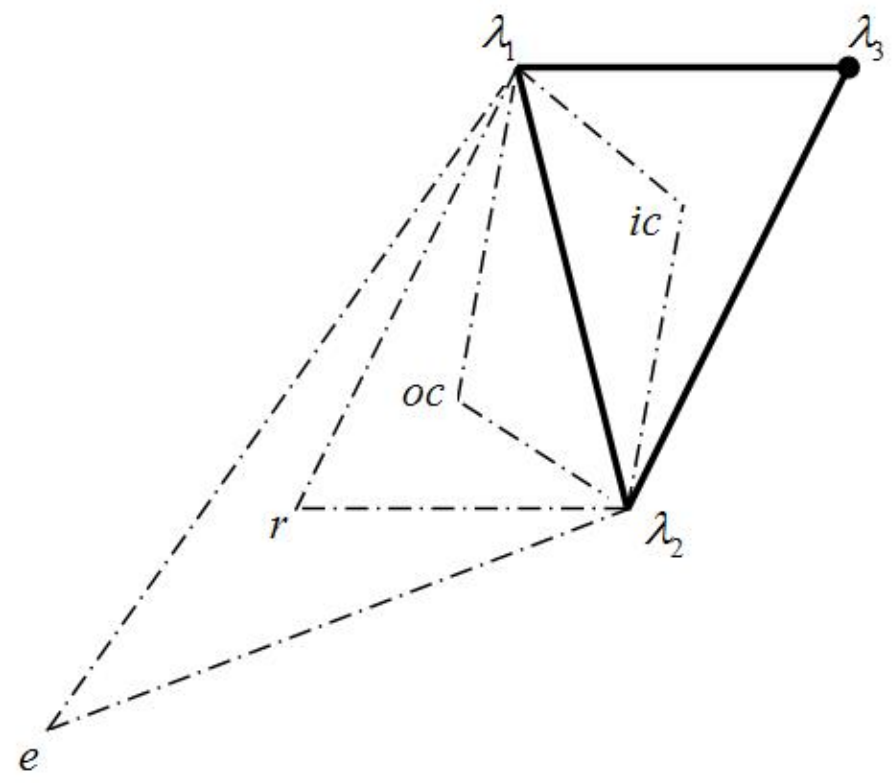

Figure 5: Nelder-Mead in 2 dimensions.

$$
f=f_{s}+\phi
$$

where $f$ is smooth and $\phi$ is a low-amplitude perturbation that is smallest near the minimum.

Given a current $\lambda$ and step size $h$, the algorithm proceeds as follows. First $f$ is sampled at the $2 N$ points in the range

$$
S(\lambda, h)=\left\{\lambda \pm h e_{i}\right\},
$$

where $e_{i}$ is the unit vector in the $i$ th coordinate direction. Let

$$
f^{*}=\min \{f(z) \mid z \in S(\lambda, h)\} .
$$

If $f^{*}>f(\lambda)$ then this is designated a failure, $h$ is reduced, and the routine iterates. If $f^{*}<f(\lambda)$ then the routine attempts to update $\lambda$ with the formula

$$
\lambda_{N E W}=\lambda-\nu H^{-1} \nabla_{h} f(\lambda)
$$




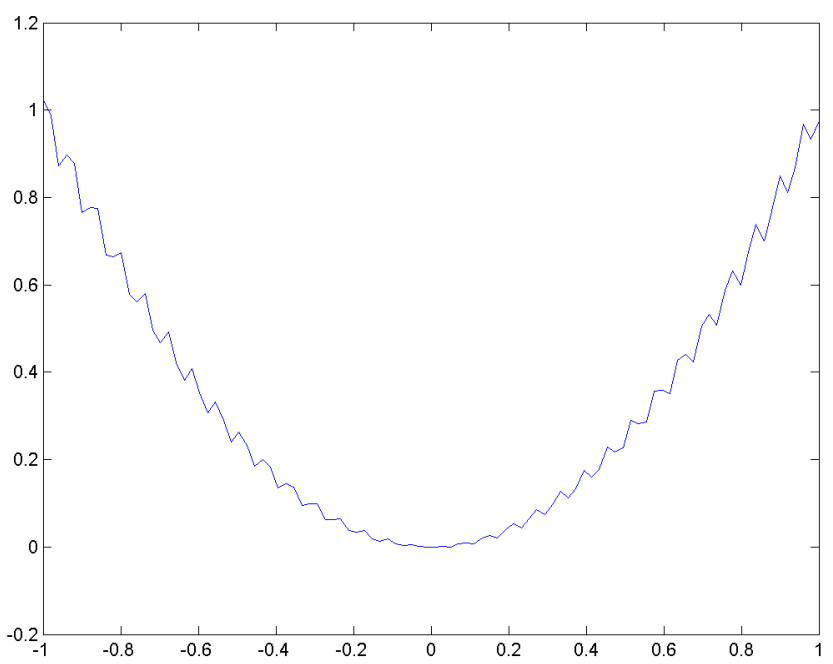

Figure 6: Example of 1D objective function in which implicit filtering would be useful.

where $H$ is the model Hessian obtained from the quasi-Newton update, $\nabla_{h} f(\lambda)$ is the approximate gradient obtained from using a centered difference formula on the available parameter range, and $\nu$ is a line search parameter to insure sufficient decrease. This proceeds until $h$ reaches a user specified value.

\section{Results}

The electron gun chosen for this research is a Pierce gun that will be used with others in a $10 \mathrm{MW}$, multiple beam klystron at L-Band. The specifications require that the beam voltage be less than $120 \mathrm{kV}$. Given the total power required and the number of guns anticipated, each gun should produce approximately $20 \mathrm{~A}$. Based on a preliminary RF circuit design, a beam fill factor of $66 \%$ was chosen as the beam size. Consequently, the goal is to develop an electron gun operating at $110 \mathrm{kV}$, producing $20 \mathrm{~A}$ with a $66 \%$ fill factor in the beam tunnel. An additional requirement is that the beam ripple be less than $5 \%$. 


\subsection{Simple Parameters (cathode radius of curvature)}

The first task was to optimize the gun performance changing simple parameters in the model. For the cathode, the only variable was the radius of curvature. This is illustrated in Figure 7 showing the SolidWorks ${ }^{\circledR}$ sketch and the associated design table. In the optimization process, the radius of curvature was modified to match the magnetic field profile at the cathode to minimize beam ripple.

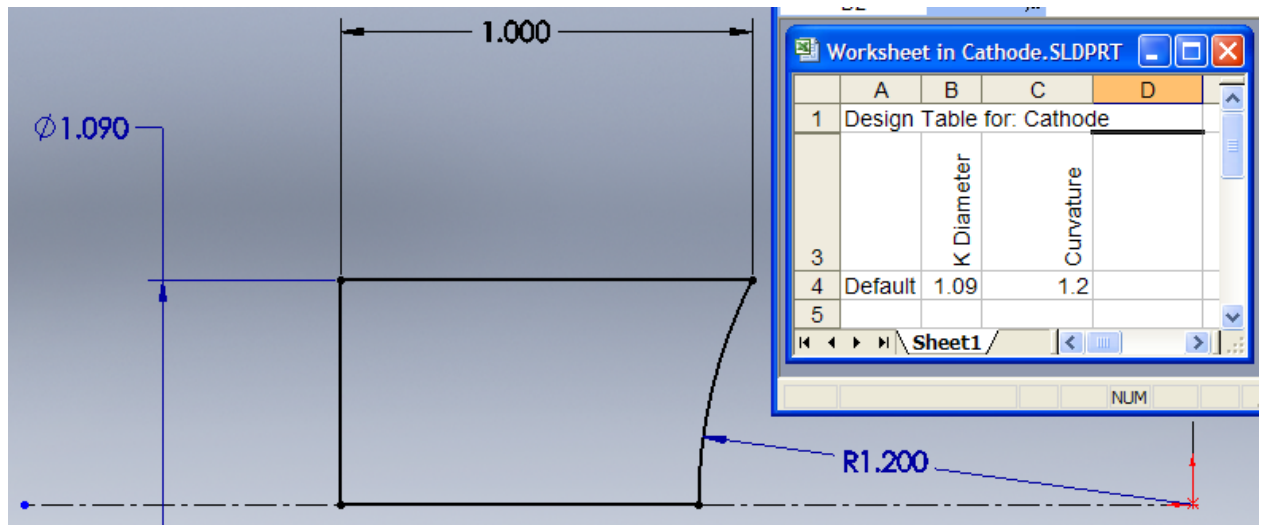

Figure 7: Sketch of cathode with associated design table.

The gun current was modified by adjusting the spacing between the cathode and the anode. This is illustrated in Figure 8, which shows a cross section of the cathode-anode region and the associated design table. Since the face of the cathode is curved, the spacing indicated is from the back of the cathode to the flat face of the anode.

The third variable was the relative position between the cathode and the magnetic circuit. Since BOA's magnetic field solver is not yet operational, the magnetic circuit was modelled in Maxwell 2D and the solution used as input to BOA. This input is part of the ASCII input file into the BOA solver, so the MATLAB ${ }^{\circledR}$ routines directly edit this file to adjust this parameter.

Evaluation of simulation results was performed in MATLAB ${ }^{\circledR}$ using output data generated by BOA. The beam current is reported by BOA directly. The beam size was evaluated from the radial coordinate of the outermost trajectory. Beam laminarity was determined as follows. Consider the radius, $r$, of each electron particle as a function of its $z$ location. Thus for each particle (disregarding the angular component, which does not affect the beam 


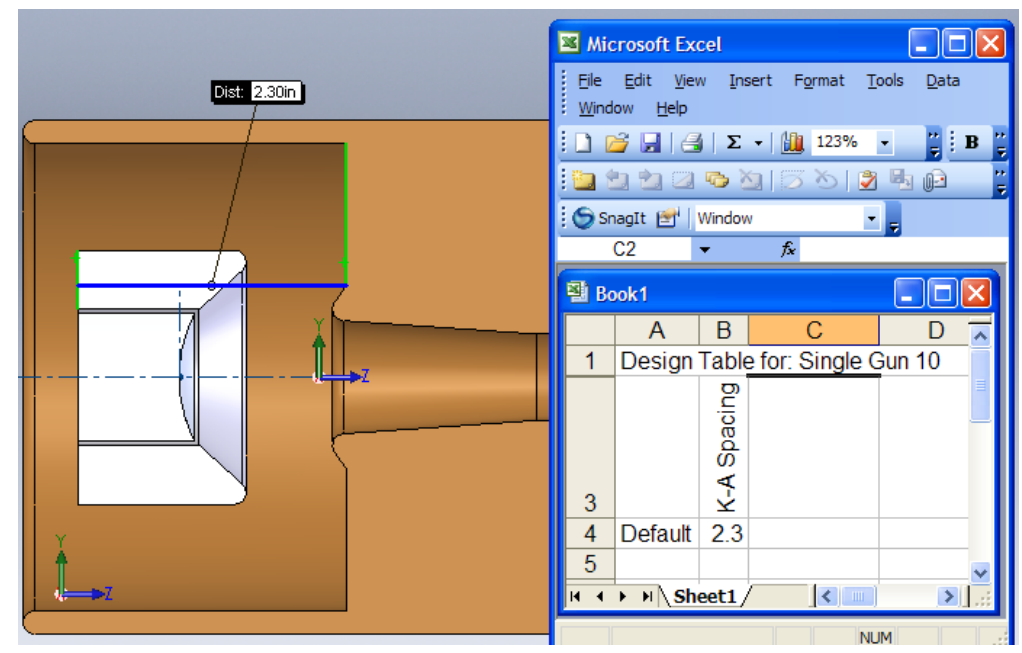

Figure 8: Cross section of the cathode-anode region with associated design table controlling cathode to anode spacing.

laminarity) one can characterize the $i t h$ particle by the function $r_{i}(z)$. Then a measure of the laminarity is the derivatives of this function with respect to $z$. However it may misrepresent the true beam shape to only look at these values at a single $z$ location, so this information is determined at several locations along the length of the beam. Since we are looking at trajectory information at specific $z$ values, we have included information about first and second derivatives of the trajectories. A zero value of the first derivative is achieved at a peak or trough in the beam shape. However the beam will be laminar at this point if the second derivative is also zero. See Figure 9 for the potential trajectories that caused us to use first and second derivative information. The goal functions that describes beam laminarity are given by

$$
\begin{aligned}
& J_{1}(\lambda)=\alpha \sum_{j=1}^{4} \sum_{i=1}^{N} \frac{\partial r_{i}\left(z_{j}\right)}{\partial z} \\
& J_{2}(\lambda)=\beta \sum_{j=1}^{4} \sum_{i=1}^{N} \frac{\partial^{2} r_{i}\left(z_{j}\right)}{\partial z^{2}},
\end{aligned}
$$

where $\alpha$ and $\beta$ are user specified weights, and $N$ is the number of particles 
used. Note the upper bound of 4 in the sum over the $j$ index is the number of $z$-locations at which this information was obtained.

The second goal is to produce a beam with a specific radius. The goal function used here is

$$
J_{3}(\lambda)=\gamma\left(b-b_{d}\right)^{2}
$$

where $b$ is the beam radius, $b_{d}$ is the desired beam radius, and $\gamma$ is a user specified weighting parameter.

Finally the beam should have a specified current. The appropriate goal function is

$$
J_{4}(\lambda)=\delta\left(I-I_{d}\right)^{2},
$$

where $I$ is the beam current, $I_{d}$ is the desired beam current, and $\delta$ is a user specified weighting parameter. The total goal function is

$$
J(\lambda)=\sum_{i=1}^{4} J_{i},
$$

where $\lambda$ are the parameters which specify gun design.

The specific numbers for this optimization are as follows. The weights $\alpha$, $\beta, \gamma$, and $\delta$ were chosen such that $J_{i}\left(\lambda_{0}\right)=1$ for $i=1, . ., 3$ and $J_{4}\left(\lambda_{0}\right)=10$, where $\lambda_{0}$ is the initial value for the design parameters. The initial value for this problem is $\lambda_{0}=(50.8 \mathrm{~mm} ., 25.4 \mathrm{~mm} ., 0 \mathrm{~mm})$, where the entries in $\lambda_{0}$ are the spacing between the cathode and the anode, the radius of curvature of the cathode and the relative position between the cathode and the magnetic circuit respectively. The desired beam radius is $b_{d}=6.2 \mathrm{~mm}$. The desired current $I_{d}=20 \mathrm{~A}$. The optimization process used the Nelder-Mead algorithm. A $2.8 \mathrm{GHz}$ Toshiba laptop running Windows XP executed the optimization routines and the CAD and simulation codes. Each iteration in the process required approximately eight minutes. Figure 10 shows the value of various design parameters during the process. Figure 11 shows the value of the various goal functions as the iterations proceed. The results of this optimization are summarized in Table 1.

As can be seen, the variables appear to reach essentially their final values after approximately 60 iterations. This required approximately eight hours.

The results are summarized in Figure 12 . In addition to the previously defined goal functions, the beam ripple or scallop is a value of interest. The beam scallop is defined as 

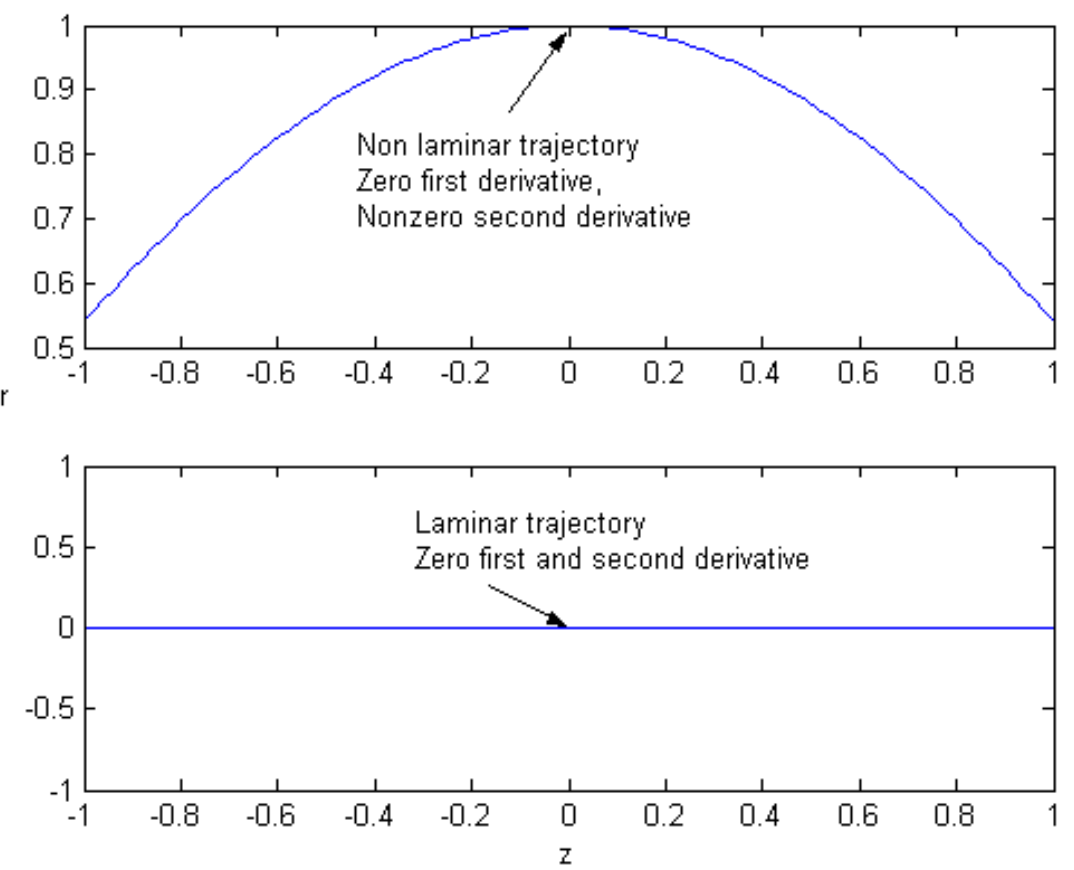

Figure 9: Examples of potential beam trajectories. 

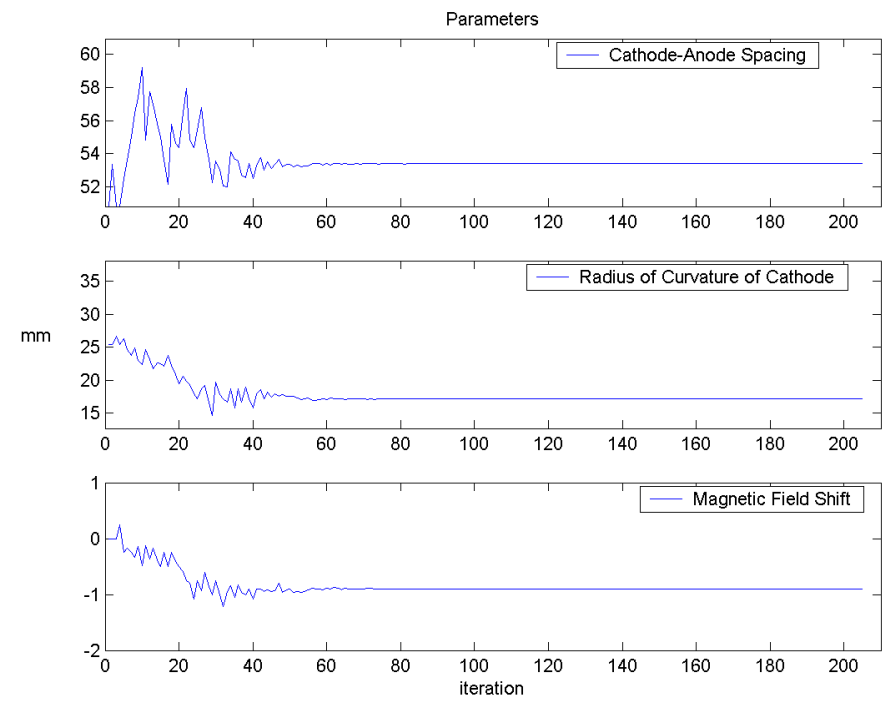

Figure 10: Design Parameters as a function of iteration.
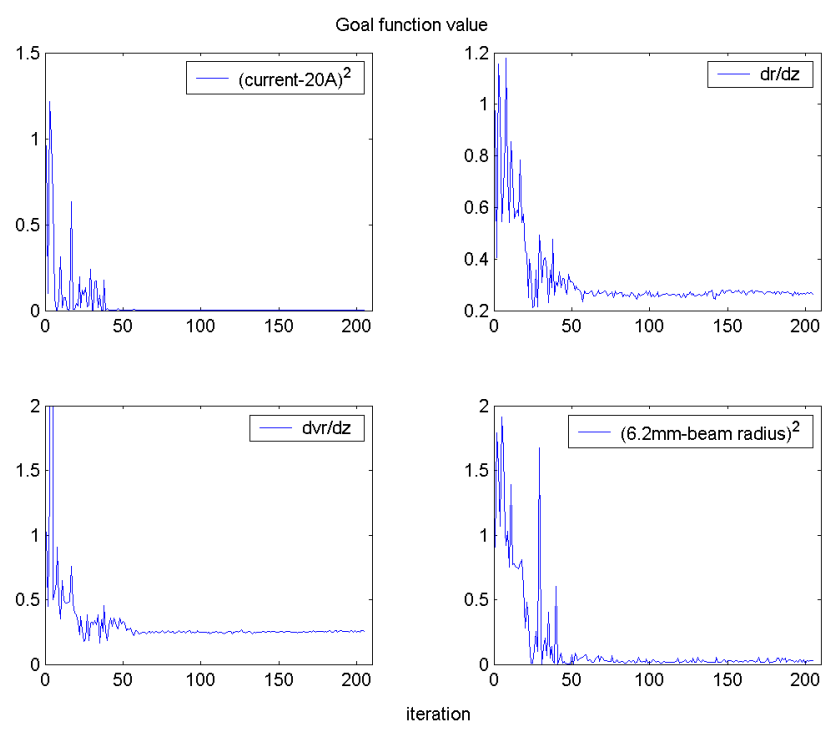

Figure 11: Goal functions as a function of iteration 


\begin{tabular}{|c|c|c|}
\hline & $\lambda_{0}$ (initial values) & $\lambda$ (optimized parameter) \\
\hline Beam Radius & $4.855 \mathrm{~mm}$ & $6.523 \mathrm{~mm}$ \\
$\frac{1}{\beta} J_{2}$ & $2.068 \mathrm{e}+022$ & $5.354 \mathrm{e}+021$ \\
$\frac{1}{\gamma} J_{3}$ & 4.662 & 1.311 \\
Current & $27.859 \mathrm{~A}$ & $19.587 \mathrm{~A}$ \\
\hline
\end{tabular}

Table 1: Performance comparison for initial optimization, where the second column indicates the performance of the gun using the initial design and the the third column indicates the performance of the gun with the optimized design parameters.

$$
\operatorname{Scall}(\lambda)=100 \frac{d_{\max }-d_{\min }}{d_{\max }+d_{\min }},
$$

where $d_{\max }$ and $d_{\min }$ denote the maximum and minimum diameter of the beam. The current was within $2 \%$ of the goal and the beam scallop was well within the goal of $5 \%$ or less. The beam size was approximately $7 \%$ from the desired value. Considering that the magnetic profile is not optimized and only three variables were available for modification, these results were very encouraging. 


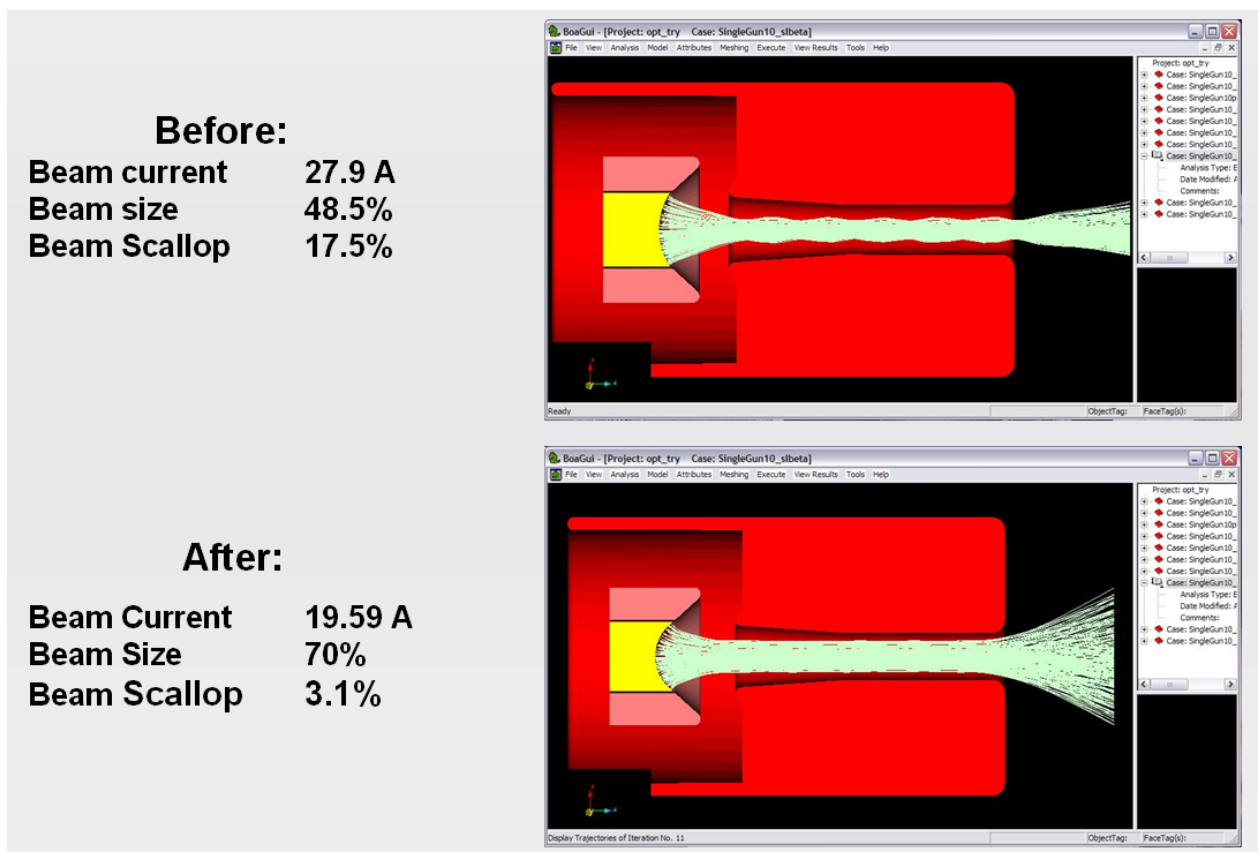

Figure 12: Optimization Results. 


\section{Spline Cathode}

It is anticipated that optimization of 3D structures will require modification of electrode shapes that can not be achieved with simple dimensional changes of lines, arcs or circles. Previous experience indicates that outstanding results can be achieved if one is not restricted to simple changes of lengths and radii $[9,10]$. The next task was to demonstrate that shape optimization could be implemented and achieve further improvements in performance.

A cathode was designed that consisted of points along a radius of the cathode connected by a spline. The full cathode was generated by rotating the curve about the cathode axis. Figure 13 shows the initial configuration. The cathode was defined by six points along a constant curvature arc from the optimization in the previous section. The optimization routines were allowed to freely change the axial position of the points, but not the radial position.

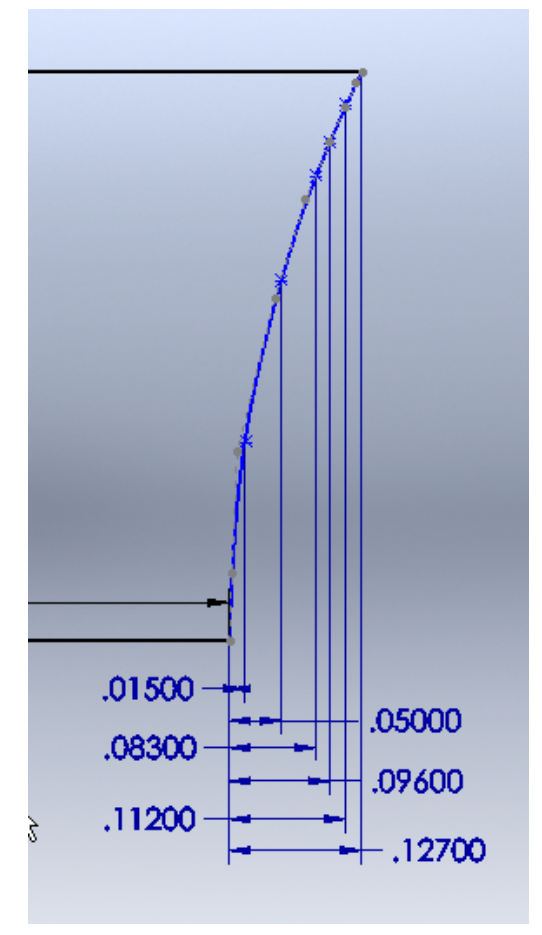

Figure 13: Spline Cathode 
For this optimization, the beam scallop was again evaluated as given by formula (14).

The goal was to minimize the function

$$
J(\lambda)=J_{3}(\lambda)+\operatorname{Scall}(\lambda) .
$$

The initial decision to vary all points of the spline proved problematic. Changes to points near the axis could not impact the value of the cost function. Thus the process failed to converge and produced unrealistic cathode shapes. Therefore, it was necessary to limit the number of points that could be varied, specifically, those near the outer edge, and constrain the range of modifications for each point. Consequently, the design engineer can not be totally eliminated from decisions necessary to achieve a complete design.

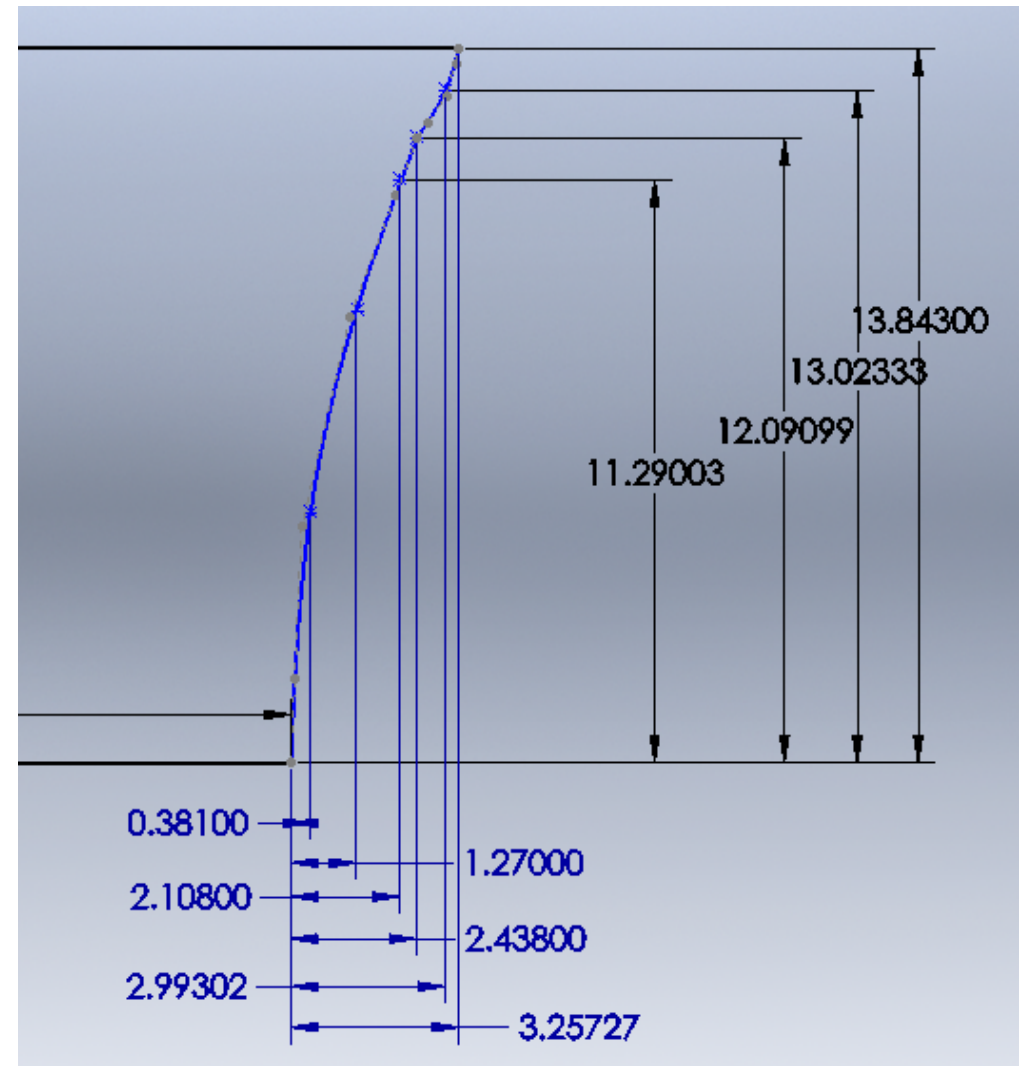

Figure 14: Sketch of cathode defined by spline curve. 


\begin{tabular}{|c|c|c|}
\hline & $\lambda_{0}$ (initial values) & $\lambda$ (optimized parameter) \\
\hline BeamRadius & $4.25 \mathrm{~mm}$ & $6.21 \mathrm{~mm}$ \\
Scall & $16 \%$ & $4.83 \%$ \\
\hline
\end{tabular}

Table 2: Performance comparison for initial spline optimization.

Only the outer two points were selected for modification, and, again, only the axial coordinate could be changed. As a constraint, the axial distance of each point from the center of the cathode could not be less than any points with a smaller radius. This would result in electrons being emitted with a positive radial velocity, which was assumed to be at variance with the desired result. The distance between the cathode and anode was fixed from the previous optimization. The relative position between the cathode and the magnetic circuit was the third parameter in addition to the two outer points on the spline cathode.

For this problem Nelder-Mead performed poorly, so the optimization routine used implicit-filtering. The optimization resulted in a beam scallop of less than $5 \%$, and the beam size was within $2 \%$ of the target value. The final cathode shape is shown in Figure 14. Note the subtle deviation from a purely spherical shape near the outer edge. The results are summarized in Table 2 and Figures 15 and 16. 


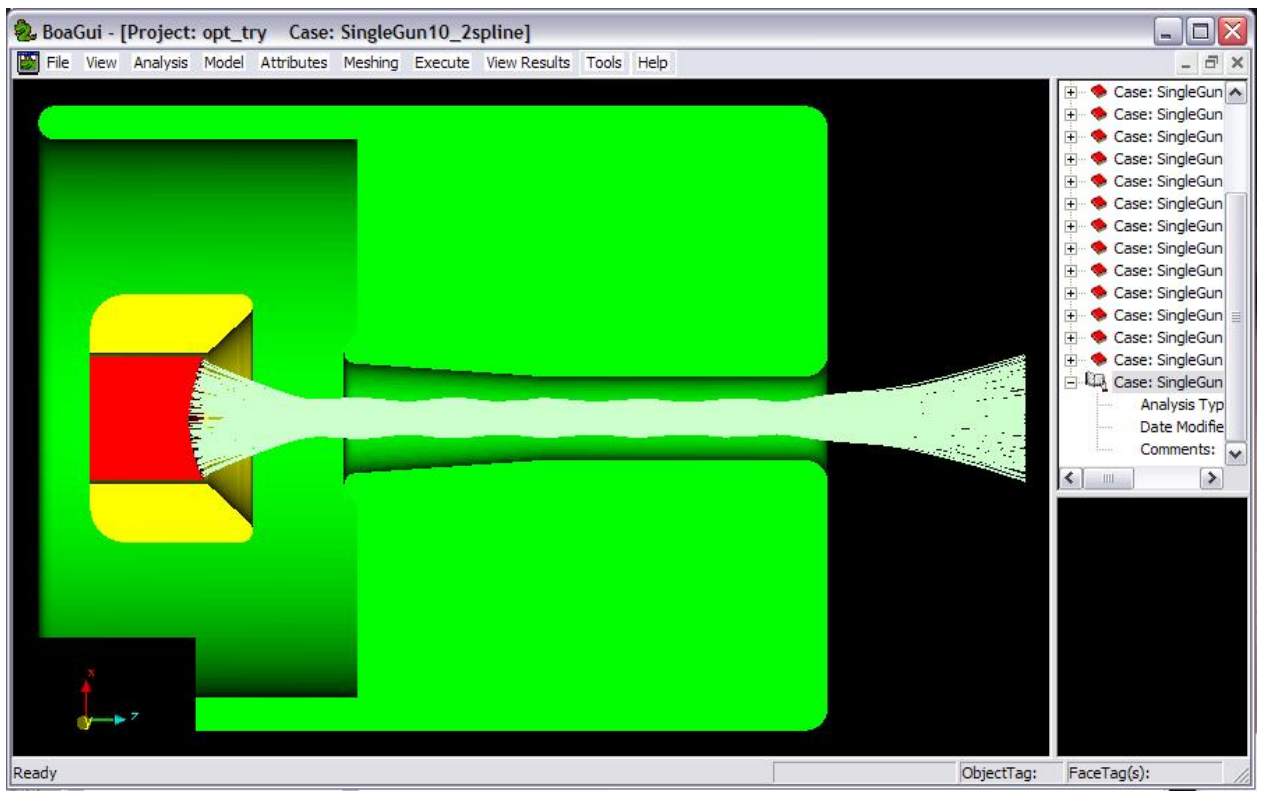

Figure 15: Spline optimization: before.

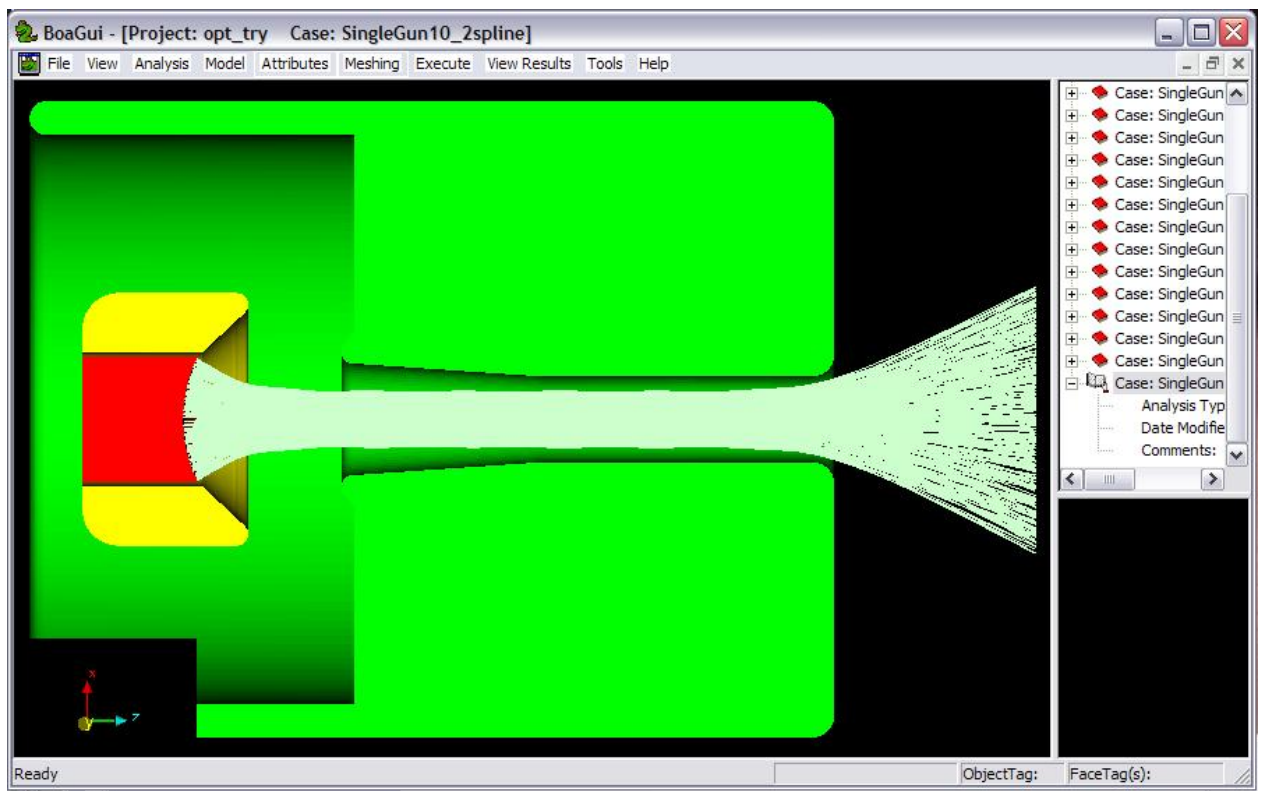

Figure 16: Spline optimization: after. 


\section{Electric Field Optimization: Spline Focus Electrode}

A key failure mechanism in electron guns is arcing between the the focus electrode and the anode. Consequently, there is a desire to minimize the electric field so long as the beam performance is not compromised. For this optimization, the focus electrode was defined by a series of points connected with a spline. As with the cathode, the final electrode was generated by rotating the curve about the axis. Figure 17 shows the starting configuration for the optimization which consisted of a straight line between the inner radius of the electrode to a constant radius arc tangent to the outer radius. From experience, it is known that high field gradients only occur in the region of the focus electrode closest to the anode. Consequently, only points in this region, specifically, points $\mathrm{P} 4$ through $\mathrm{P} 6$, were selected for optimization. The optimization routines were allowed to change the axial position of the points, but not the radial position. Constraints were added to prevent generation of unrealistic configurations.

BOA can calculate electric field vectors at discrete points on the surfaces. The electric field vector on the focus electrode was denoted by $E_{i}$. The goal function for this problem was

$$
J(\lambda)=\max \left\|E_{i}\right\| .
$$

Note that since the goal function is the maximum value of the electric field on the focus electrode and we are trying to minimize this goal function, we are thus minimizing the maximum value of the electric field in the device. Using the Nelder-Mead algorithm, the magnitude of the vector was reduced for $J$ by $6.7 \%$. Figure 18 shows the final shape from the optimization process.

Recall that only points P4 through P6 were modified during the optimization process. The position of $\mathrm{P} 3$ was not allowed to change, which explains the curvature in the profile at this location. While this shape satisfies completely the optimization goal, it could present unnecessary complications for machining. Using engineering experience and knowing that slight changes in this region would not significantly impact performance, the location of P3 was manually modified to remove the slight bump in the profile. This is another indication that engineering experience and knowledge are important in any computational design effort. The resultant change in the maximum electric field was less than .008\%. This final configuration is shown in Figure 


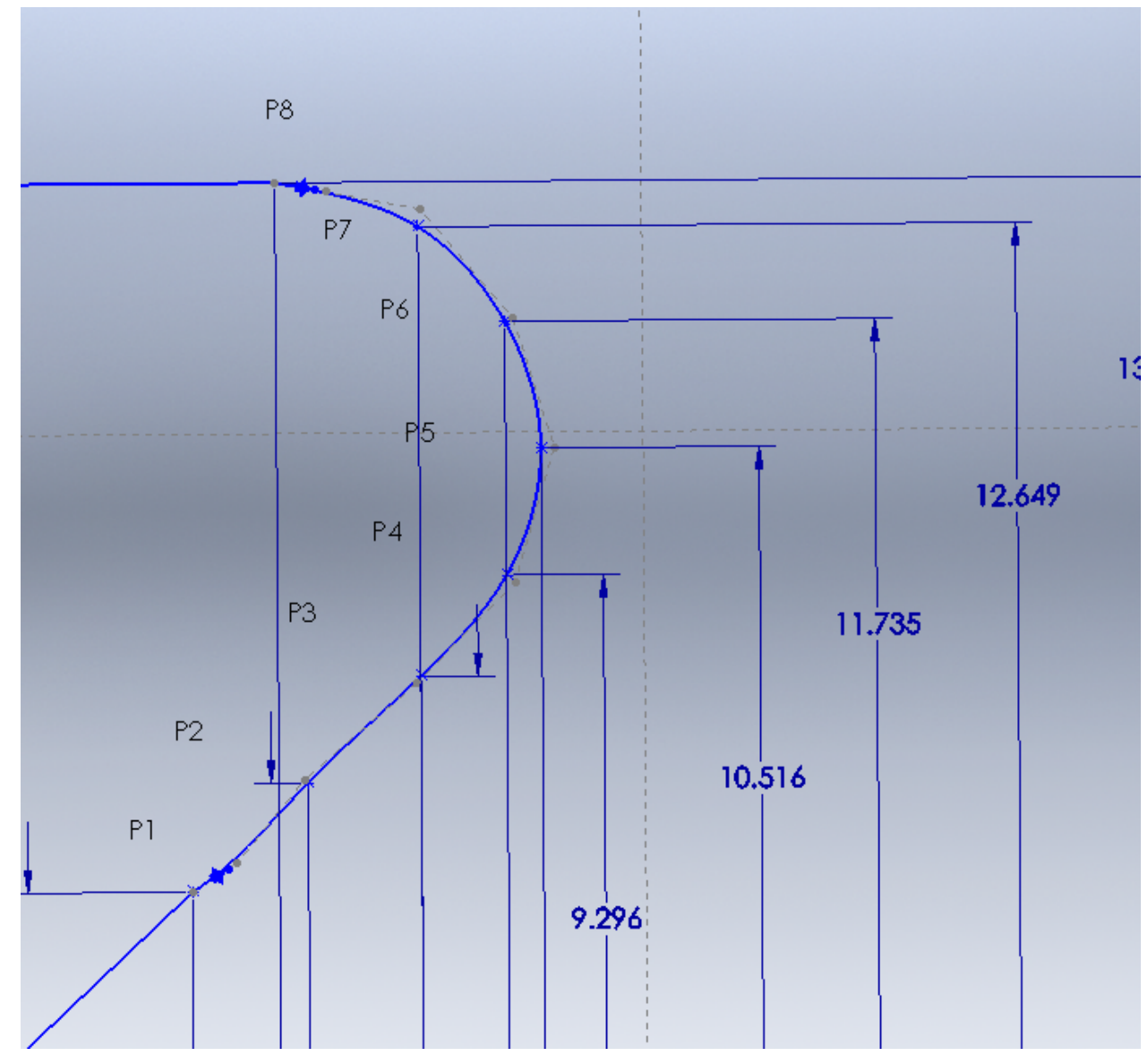

Figure 17: Initial focus electrode. Note that the dots along the focus electrode are the values specified by the user for the shape. The dark line is then the spline created from these points. The dashed lines specify other aspects of the CAD drawing. 


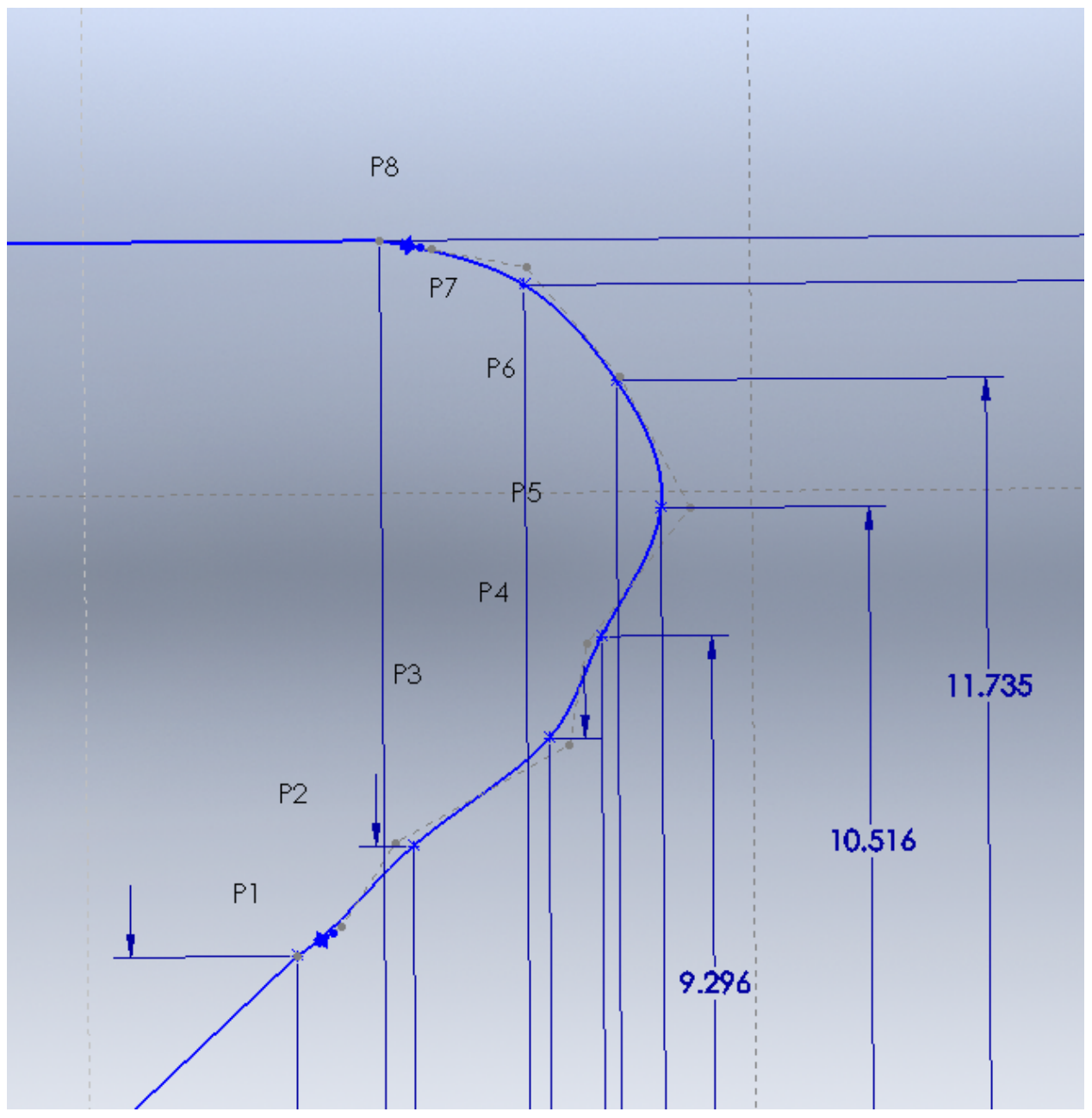

Figure 18: Optimized focus electrode. 
19. The final task was to ensure that the focus electrode modification was still compatible with the desired beam performance. The full beam simulation was performed, and the change in beam diameter was negligible, and the beam scallop changed from $4.8 \%$ to $5.1 \%$. As this violated our goal of under $5 \%$ scallop, we reran an implicit filtering optimization similar to that described in Section 6 with the focus electrode found in this section. Using this routine we were able to reduce the beam scallop to $4.4068 \%$. 


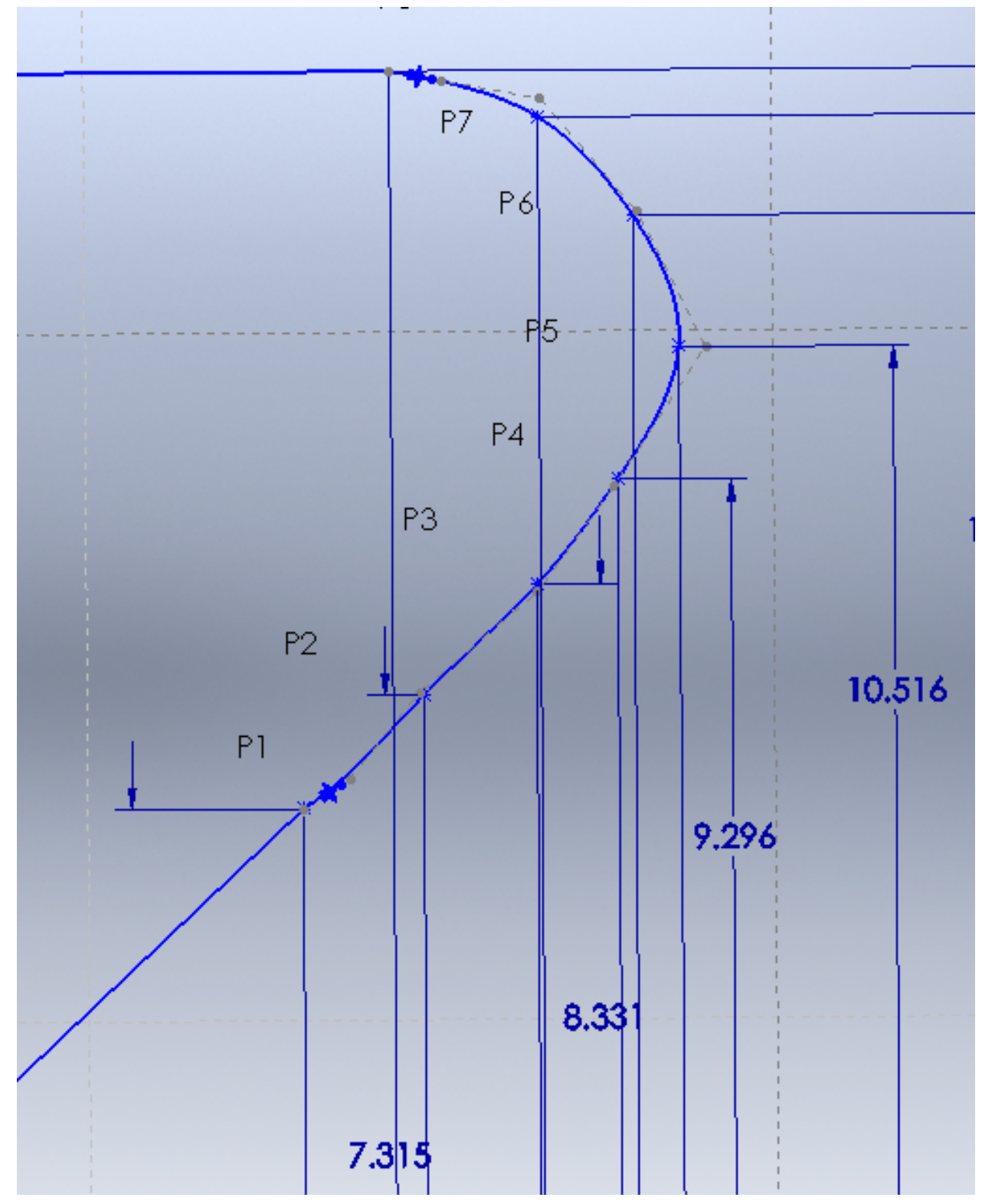

Figure 19: Final focus electrode. 


\section{Planned 3D Design}

The ultimate goal of this research is to develop design tools for complex, 3D, electron beam devices that provide improved capabilities or performance over standard 2D devices. In the microwave tube industry, significant research is under way to utilize distributed beam devices, including sheet beam and multiple beam configurations. Distributed beams allow significant reduction in operating voltage, which translates to dramatically lower cost power supplies and improved operating efficiency. It is also anticipated that a new generation of electron devices could be developed for new and innovative applications.

\subsection{Sheet beam gun}

Several organizations, including CCR, are developing sheet beam RF sources. These have lower operating voltage, improved efficiency, greater bandwidth, and reduced fabrication cost. They are being developed for the International Linear Collider, which will require several hundred klystrons. Therefore, the potential impact is quite large.

Sheet beam cathodes typically use a cylindrical emitter to produce the rectangular electron beam. The beam is compressed in only one dimension. A major issue is the design of the corners of the electron gun, where electric fields are abruptly changing. Since 3D analysis is required, iterative design is particularly time consuming. Not only does the beam simulation take significant time, but manual modification of the 3D geometry itself can also be labor intensive.

Modern solid modelling programs can model 3D surfaces defined by points in space. These points can be defined by dimensions relative to a common origin and, therefore, included in design tables. The initial effort will be to modify the shape of the cathode in the corners and the adjacent focus electrode. Simple modifications will be performed initially to verify proper operation of the process and better understand the relationship of various parameters on beam performance. Figure 20 shows one quarter of the sheet beam cathode with its associated design table. Note that the location of the corner spline point was moved forward, causing the corner of the cathode to be pointed more toward the axis of the device.

Similarly, the shape of the adjacent focus electrode can be manipulated to modify the electric fields accelerating the electrons near the cathode. The 


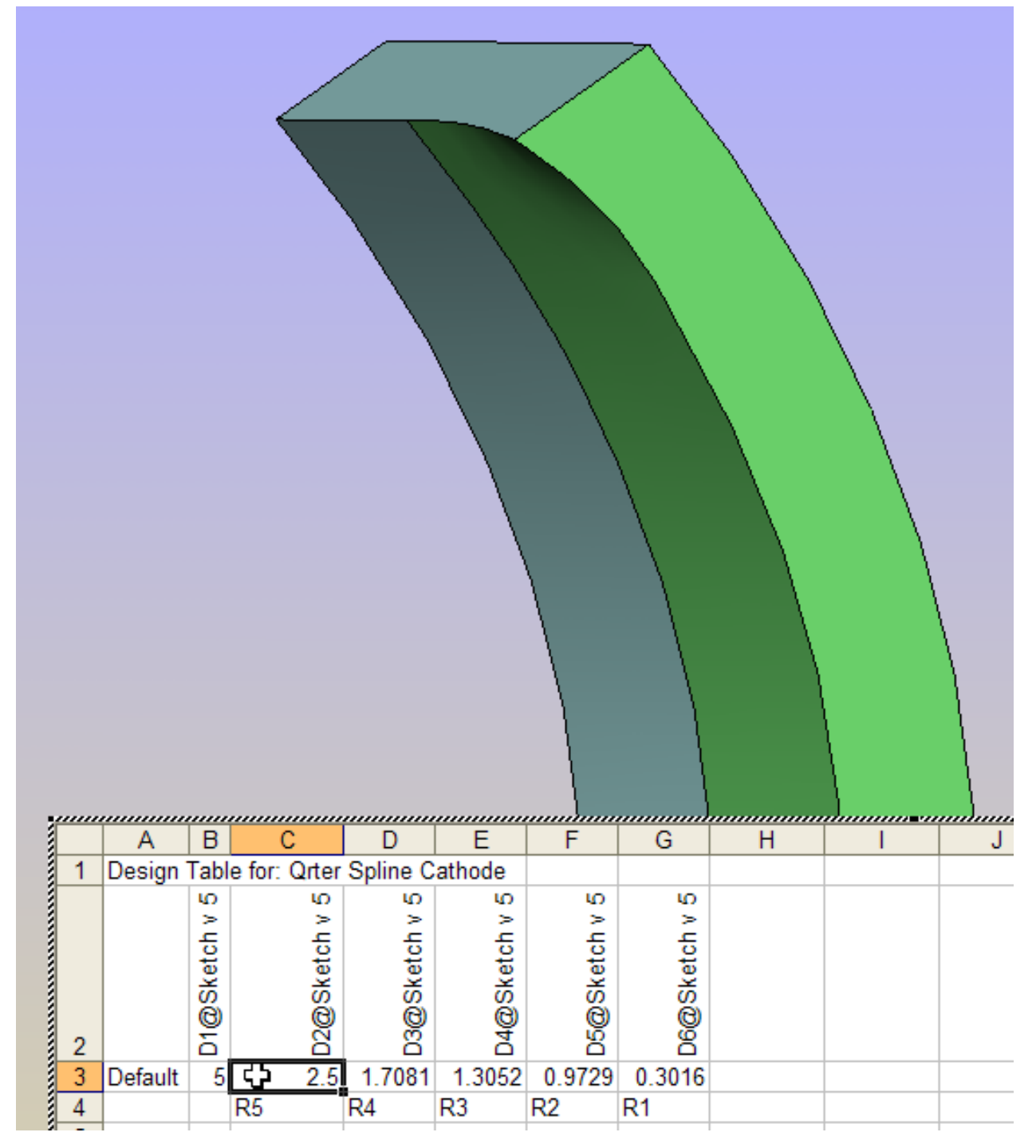

Figure 20: Quarter of Sheet Beam Gun with parameter table. 
initial effort will be to modify the shape of the cathode and focus electrode to properly focus corner electrons.

A challenge for the sheet beam gun optimization will be defining a goal function to evaluate the performance. It will be necessary to identify the corner trajectories and generate a numerical value for the 'quality' of these electron trajectories. Since the number of trajectories and their specific emission location can vary from geometry to geometry, innovative evaluation techniques will be required. This will be a key challenge in the upcoming research.

\subsection{Multiple beam guns (MBGs)}

In 2005, the U.S. Department of Enegy awarded CCR a contract to develop a doubly convergent MBG for an L-Band klystron. The current generation of multiple beam electron guns use singly convergent beams. This means the beams converge about their individual axes, defined by the cathodes, but do not converge toward the axis of the device. Design of MBGs that converge about the beams' local axis and the device axis are referred to here as doubly convergent MBGs. After several months of development, it became apparent that the number of variables exceeded capabilities to manually design such a device. Consequently, the effort was abandoned, and CCR initiated the current program to develop computer optimization techniques for such 3D devices. As the individual beams converge about their local axis, they begin to rotate about that axis to create the $v \times B$ force necessary to balance space charge forces within the beam. Nevertheless, the beams continue to propagate parallel to the device axis in singly convergent guns. For doubly convergent beams, the compression toward the device axis causes the beams to rotate about the axis of the device, following a spiral

path. This spiraling about the device axis makes design and fabrication of complex circuits impractical, at least using currently available fabrication techniques.

The rotation of doubly convergent MBG beams about the device axis is necessary to conserve angular momentum. If the electrons could be emitted from the cathodes at an angle to the device axis (and magnetic field), they could possibly satisfy conservation requirements with the beams propagating parallel to the device axis when fully compressed. Angular injection at the cathode will require complex, 3D structures to preserve the beam quality. Following completion of the sheet beam gun effort, CCR will use optimization 
techniques to design these complex structures.

Successful development of doubly convergent MBGs would allow design of high power, multiple beam klystrons using fundamental mode circuits. Existing multiple beam, high power devices must use overmoded circuits to avoid excessive emission current densities at the cathode. Not only does this complicate the design, but increases the radial size and potential for parasitic mode generation. The increased size of the device also increases the size of the magnetic circuit and, hence, its power supply requirements.

\section{Summary}

The 2D research demonstrated that computer optimization could be effectively applied to electron gun design. Using the procedures established in this program, it should be possible for a design engineer to set up an optimization process achieving specified electron gun voltage, current, beam size, and beam ripple goals in a few hours . Compared to the 20-30 hours typically required with current, manual design, this is more than an order of magnitude reduction in engineering design time. Greater savings are anticipated with more complex designs with additional variables, including designs for $3 \mathrm{D}$ devices.

Additionally, computer optimization can explore significantly larger parameter space than can be practically accomplished manually. Manual techniques for simple Pierce guns typically simulate 20-30 variations of the design. The computer optimization processes in this program explored 50-60 variations before determining the optimum configuration. As demonstrated in previous optimization efforts $[11,12,13,14,15,16]$, computer designs typically exceed the performance achieved with manual design.

If $3 \mathrm{D}$ optimization techniques can be successfully developed, it will enable a new generation of devices that can not currently be designed. This will allow new innovative applications for electron beam devices.

\section{Acknowledgement}

This research was funded by U.S. Department of Energy Small Business Innovative Research Grant DE-FG02-06ER86267. 


\section{References}

[1] M. Cattelino, "Communications and power industries," private communications, December 2006.

[2] M. Read, V. Jabotinski, G. Miram, and L. Ives, "Design of a gridded gun and ppm-focusing structure for a high-power sheet electron beam," IEEE Transactions on Plasma Science, vol. 33, no. 2, pp. 647-653, April 2005.

[3] R. L. Ives, G. Miram, and A. Krasnykh, "Electron gun for multiple beam klystron using magnetic focusing," Patent, 2004.

[4] "Development of a $200 \mathrm{MHz}$ multiple beam klystron," Calabazas Creek Research, Inc., Tech. Rep. U.S. Department of Energy Grant DE-FG0204ER83916, 2005.

[5] A. Gilmour, Jr., Principles of Traveling Wave Tubes. Artech House.

[6] B. Lewis, H. Tran, M. Read, and L. Ives, "Design of an electron gun using computer optimization," IEEE Transactions on Plasma Science, vol. 32, no. 3, pp. 1242-1250, June 2004.

[7] C. T. Kelley, Iterative Methods for Optimization. SIAM, 1999.

[8] - "A brief introduction to implicit filtering," CRSC, Tech. Rep. CRSC-TR02-28, 2002.

[9] R. Ives, J. Neilson, and W. Vogler, "Cascade - An advanced computational tool for waveguide system and circuit design," IEEE Intern. Conf. on Plasma Sci., 1998.

[10] J. Neilson, "Optimal synthesis of quasi-optical launchers for high power gyrotrons," IEEE Transactions on Plasma Science, vol. 34, no. 3, June 2006.

[11] D. Abe, T. Antonsen, Jr., D. Whaley, and B. Danly, "Design of a linear C-band helix TWT for digital communications experiments using the CHRISTINE suite of large-signal codes," IEEE Transaction of Plasma Science, vol. 30, no. 3, pp. 1053-1062, 2002. 
[12] D. Whaley, C. Armstrong, B. Gannon, G. Groshart, E. Hurt, J. Hutchins, and M. Roscoe, "Sixty-percent-efficient miniature C-band vacuum power booster for the microwave power module," IEEE Transaction of Plasma Science, vol. 26, no. 3, pp. 912-921, 1998.

[13] J. Neilson and R. Ives, "Computer optimization and statistical tolerancing analysis of waveguide windows," in International Vacuum Electronics Conference, Monterey, CA, 2000.

[14] A. Singh, S. Rajapatirana, Y. Men, V. Granatstein, R. Ives, and A. Antolak, "Design of a multistage depressed collector system for 1-MW CW gyrotrons. I. trajectory control of primary and secondary electrons in a two-stage depressed collector," IEEE Transactions on Plasma Science, vol. 27, no. 2, pp. 490-502, 1999.

[15] L. Ives, J. Neilson, and W. Vogler, "Rapid waveguide system and component design using scattering matrices and computer optimization," in IEEE International Conference on Plasma Science San Diego, CA, USA, 1997, p. 160.

[16] W. Lawson, M. Arjona, B. Hogan, and R. Ives, "The design of serpentine-mode converters for high-power microwave applications," IEEE Transactions on Microwave Theory and Techniques, vol. 48, no. 5, pp. 809-814, 2000. 